\title{
Evaluation of a large-scale tuberculosis contact investigation in the Netherlands
}

\author{
K. Borgen*,\#, B. Koster`, H. Meijer', V. Kuyvenhoven ${ }^{\S}$, M. van der Sande ${ }^{\#}$ \\ and F. Cobelens ${ }^{\S, f}$
}

ABSTRACT: The aim of the present study was to evaluate yield and effectiveness of a large-scale contact investigation around a supermarket employee with infectious tuberculosis.

Supermarket customers were screened by tuberculin skin test (TST) and/or radiography, depending on individual characteristics. The number of recent infections was estimated based on historical reference data after correction for false-positive TST results.

TST screening of 15,518 subjects yielded 12 cases of tuberculosis disease as a direct result of the investigation (1,293 screenings per case identified). Radiographical screening of 5,945 subjects yielded no cases. There were $359(2.6 \%)$ positive TSTs; $117(34 \%)$ were estimated to be due to recent exposure. The number of customers screened in order to find one case of recent infection was 114, varying from 43 for customers who visited the supermarket twice per week or more, to 4,148 for customers who visited less than once per month.

In conclusion, although this patient probably transmitted Mycobacterium tuberculosis to at least 117 customers, the contact investigation was inefficient, as large numbers of customers had to be screened and the majority of identified tuberculosis infections were probably not related to the index case. The efficiency could have been improved by omitting radiographical screening and limiting tuberculin skin test screening to customers who reported frequent supermarket visits.

KEYWORDS: Control, efficiency, public health, tuberculosis

$\mathbf{T}$ uberculosis (TB) remains a major cause of morbidity and mortality worldwide [1]. Many industrialised countries have been able to implement effective control policies and thereby reduce the incidence to very low levels. Apart from treatment of passively detected cases, TB control in low-prevalence settings focuses on active case detection among high-risk groups and contacts of infectious TB cases, and identification and preventive treatment of latent TB infection (LTBI) [2-4]. These investigations are generally limited to household and other close (e.g. workplace) contacts [5-9]. A high prevalence of infection or multiple cases of disease suggests unusual bacterial virulence or duration of infectiousness of the source case, and may be reason to expand the investigation to a larger number of casual contacts [9-11]. An important question when expanding $\mathrm{TB}$ investigations is how wide the net should be cast. The more casual contacts included, the more infections will be detected, but there will also be more false-positive tuberculin skin tests (TSTs) and remote infections unrelated to the source case with low probability of progression to disease [12].
In the Netherlands, the incidence of $\mathrm{TB}$ over the past $10 \mathrm{yrs}$ has been $<10$ per 100,000 of the population. There is no routine bacille CalmetteGuerin (BCG) vaccination programme and the estimated prevalence of infection is $<5 \%$ in the indigenous Dutch population born after 1950 [13]. This makes the TST a suitable tool for detection of recent LTBI in contact investigations in the Dutch-born population [14, 15]. Among the foreign-born inhabitants, BCG vaccination is frequent and the prevalence of infection is higher; therefore, TST screening of foreign-born contacts is not routine. Contact investigations are performed by Municipal Health Services (MHS) responsible for TB control, according to the "stone in the pond principle" [10]. Contacts are defined in concentric circles around the source case, depending on the frequency and intimacy of their contact. Individuals belonging to the innermost circle are investigated by TST and/or chest radiograph (CXR), depending on their age, BCG vaccination status and country of birth. If the observed prevalence of infection is deemed considerably above the expected value, the investigation is extended to the next circle until the prevalence of
AFFILIATIONS

*European Programme for

Intervention Epidemiology Training (EPIET),

${ }^{\#}$ Dept of Infectious Disease Epidemiology, National Institute for Public Health and the Environment (RIVM), Bilthoven,

"Municipal Health Service (GG and GD) Utrecht, Utrecht,

${ }^{+}$Municipal Health Service (GGD) Midden-Nederland, Zeist,

${ }^{5}$ KNCV Tuberculosis Foundation, The Hague, and

${ }^{f}$ Center for Infection and Immunity Amsterdam (CINIMA), Academic Medical Center, Amsterdam, The Netherlands.

CORRESPONDENCE

F. Cobelens, KNCV Tuberculosis

Foundation, Parkstraat 17, 2514 JD

The Hague, The Netherlands.

Fax: 31703584004

E-mail: cobelensf@kncvtbc.nl

Received:

October 162007

Accepted after revision:

March 012008

\section{SUPPORT STATEMENT}

The present study received financial support from the Municipality of Zeist, the Netherlands. Part of these data were presented at the 36th International Union Against Tuberculosis and Lung Disease (IUATLD) conference in Paris, France October 18-22, 2005 (abstract number PC-1468-21), and the 10th European Programme for Intervention Epidemiology Training (EPIET) Scientific Seminar, Mahon, Minorca, Spain, October 13-15, 2005 (abstract number 20050090).

STATEMENT OF INTEREST

None declared.

European Respiratory Journal Print ISSN 0903-1936 Online ISSN 1399-3003 
infection in the subsequent circle is not expected to be above the age-specific background prevalence [13]. In the Netherlands, large-scale contact investigations are not infrequent. From 19942004, 31 investigations that involved $>1,000$ individuals per investigation were performed. There have been no published evaluations of the effectiveness of these investigations and of their contribution to TB control in low-prevalence settings.

In 2005, a large-scale contact investigation, including over 21,000 customers of a supermarket in the Netherlands, was carried out after an employee with cavitary TB had infected a large proportion of close contacts and co-workers. The present study describes this investigation and evaluates its yield and effectiveness, in order to advise on future approaches in similar situations.

\section{METHODS}

\section{Study population}

In November 2004, a 25-yr-old male, Dutch-born, full-time supermarket employee in Zeist (a city with $\sim 60,000$ inhabitants), the Netherlands, was diagnosed with sputum smearpositive cavitary TB after a 12-month history of cough. Of 12 close contacts, three $(25 \%)$ were diagnosed with active TB and seven (58\%) with LTBI. Among 80 supermarket co-workers subsequently examined, TB infection was diagnosed in 47 $(59 \%)$, including one case of TB disease. Mycobacterium tuberculosis isolates were shown to have the same IS6110 restriction fragment length polymorphism (RFLP) pattern as the index case. These results indicated that the source patient was highly infectious or had been infectious for a long period.

In early December 2004, the local authorities decided to expand the investigation to include the customers of the supermarket. The number of people eligible for investigation was estimated to be $\sim 23,700$ inhabitants, based on a telephone survey in an area of $4.4 \mathrm{~km}^{2}$ around the supermarket. An invitation letter was posted to all households in this area, announcing the planned investigation among individuals who had visited this supermarket between January 1 and November 18, 2004. Customers living outside the identified area were invited through locally distributed flyers, the internet and press releases.

The investigation took place during 1 week in early February 2005 in a local sports hall in Zeist. Participants completed a short, self-administered questionnaire on their demographic information, TB and BCG history, previously positive TST and presence of any immunocompromising condition (defined as organ transplant, HIV infection or use of immunosuppressive medication). Since the questionnaire had to be limited in size and customers tend to visit supermarkets at regular intervals, exposure was categorised by frequency of visits to the supermarket. An anteroposterior CXR was performed on all individuals born before 1945 or with a history of BCG vaccination after the first year of life, TB or positive TST. All other individuals had a TST according to the Mantoux method, using 2 tuberculin units (TU) of RT23 in Tween 80 (Statens Serum Institute, Copenhagen, Denmark). This tuberculin is bioequivalent to the international standard of 5 TU purified protein derivative-S [16]. Skin reactions were read after 72-120 h [17].
In the present study, definitions used were as follows. TB infection (TBI): TST $\geqslant 15 \mathrm{~mm}$, or TST $\geqslant 5 \mathrm{~mm}$ if immunocompromised or $<5$ yrs of age (in accordance with Dutch guidelines [18]), with or without TB disease; LTBI: TBI in the absence of TB disease; culture-confirmed TB: CXR findings or symptoms suggestive of active TB and a positive culture for M. tuberculosis; nonculture-confirmed TB: CXR findings, with or without symptoms, suggestive of active TB, with negative or no culture results for $M$. tuberculosis.

Participants diagnosed with TBI or suspected TB disease were referred for follow-up at the MHS in Utrecht (the Netherlands). Follow-up consultations involved clinical examination, a CXR, sputum collection and referral for further diagnostic evaluation if indicated. Mycobacterial culture was performed on clinical materials. The M. tuberculosis isolates were DNA fingerprinted by IS6110 RFLP typing at the National Institute for Public Health and the Environment (Bilthoven, the Netherlands), according to standard procedures $[19,20]$. Participants diagnosed with LTBI were offered a 6-month course of isoniazid preventive therapy (IPT) or 6-monthly follow-up examinations, including CXRs, for 2 yrs, depending on age, co-medication and individual preference. Individuals not identified with TBI but with a TST reaction size of 10-14 $\mathrm{mm}$ were later invited for CXR examination. Initial diagnoses of TB with no or negative culture results were reviewed by an independent TB specialist. The yield of TB was classified, by three of the present authors not involved in the contact investigation, as direct yield of the contact investigation if detected as a result of the initial CXR screening with or without additional investigations performed due to TBI or a TST result of 10-14 mm. Cases of TB identified during IPT were classified as indirect yield.

\section{Data analysis}

Individuals with a missing TST or CXR result were excluded from the analyses. Associations between TBI, frequency of visits to the supermarket and other variables were analysed by univariate and multivariate logistic regression [21].

For the evaluation of yield and effectiveness of detection of LTBI, the proportion of detected TBI cases considered recently acquired was estimated, i.e. those attributable to infection in the supermarket. Historical reference data based on the infection prevalence measured among Dutch military conscripts from 1910-1980 were used. Applying the estimated annual risk of infection as it decreased over time, these prevalence data were extrapolated to yield the expected prevalence of TBI per 10-yr age group in 2005 [13]. Expected prevalence values for age groups born after 1960 were extrapolated assuming continuing proportional decline of the annual risk of infection. As the historical data had been collected using 1 TU RT23 or a bioequivalent tuberculin at a cut-off of $10 \mathrm{~mm}$, these expected age-specific prevalences of TBI in 2005 were adjusted for the difference in sensitivity from that of 2 TU RT23 and cut-off $15 \mathrm{~mm}$, which was used in the present contact investigation [22]. The resulting prevalence values were considered to represent the age-stratified background prevalence of remote TBI in the Dutch-born population, i.e. acquired in the distant past. The prevalence of recently acquired TBI was calculated as the difference between the observed prevalence of TBI and the background prevalence of remote TBI. Among the participants considered to have recent 
TBI, the number of false-positive TSTs, e.g. due to crossreaction with atypical mycobacteria, was estimated for each category of exposure, based on the sensitivity and specificity of 2 TU RT23 observed previously in the Dutch population [15]. From the resulting number of true positive TSTs, the following were calculated: the prevalence of recent TBI; the incremental yield in number of recent TBI cases by category of exposure, in diminishing order; and the number of people who needed to be tested in order to detect one case of recent TBI.

To check the validity of these estimates, the proportion of recent infections was also estimated based on the reported frequency of visits to the supermarket, assuming that the additional risk of infection for individuals who had visited the supermarket on average less than once a month (the baseline category) was equal to zero. The attributable risk (AR) for the other categories of frequency of visits to the supermarket was calculated as follows:

$$
\mathrm{ARi}=(\mathrm{OR} \mathrm{i}-1) / \mathrm{OR} \mathrm{i}
$$

where ARi is the AR for the frequency category $i$, and ORi is the adjusted odds ratio (OR) of TBI for the frequency category i, compared with the baseline category [23].

\section{RESULTS}

In total, $\sim 15,000$ people were estimated to be eligible for screening. Eventually, 21,326 individuals were registered in February 2005 (fig. 1). The median (range) age was 37 (098) yrs, $44 \%$ were male, and $83 \%$ stated Zeist as their place of residence. The Netherlands was reported to be the country of birth for 18,811 (88\%) individuals, with 104 other countries reported by 2,224 individuals. The country of birth remained unknown for 291 subjects.
A TST result was available for 13,970 (90\%) out of 15,518 individuals (table 1$)$. Of these, $359(2.6 \%)$ were diagnosed with TBI and investigated further, resulting in 11 cases of TB disease (fig. 1). The remaining $348(2.5 \%)$ individuals were classified as having LTBI; of these, 154 (44\%) received IPT, 122 (35\%) were assigned to CXR follow-up and $72(21 \%)$ did not report after diagnosis. Later, three $(0.9 \%)$ cases of TB disease were diagnosed among the LTBI patients during IPT (fig. 1). Of the 274 individuals with a TST of $10-14 \mathrm{~mm}$ invited later for CXR examination, 206 (75\%) reported for the examination. Of these, one $(0.5 \%)$ individual was diagnosed directly with TB disease (fig. 1), one (0.5\%) was started on IPT and $157(76 \%)$ were assigned to CXR follow-up.

Of the 5,811 individuals with a result from the initial CXR examination, $444(7.6 \%)$ had abnormal findings that warranted clinical evaluation. None of these were diagnosed with TB disease.

Thus, 15 cases of TB disease were identified as direct or indirect results of this contact investigation (table 2). All 15 were born in the Netherlands, six were male and the median (range) age was 31 (2-58) yrs. None reported an immunocompromising condition, any relationship with each other or contact with the index case, except during supermarket visits. Visiting the supermarket at least twice a week was reported by eight $(53 \%)$ of the cases, on average at least once a week by four $(27 \%)$ cases. Positive cultures of $M$. tuberculosis were obtained for seven cases; an RFLP pattern was obtained from six cases, and for five of these $(83 \%)$ the RFLP pattern was identical to that of the index case. Of the TB disease cases, 12 were classified as direct yield of the contact investigation (equivalent to 56 per 100,$000 ; 95 \%$ confidence interval (CI) 31-101 per

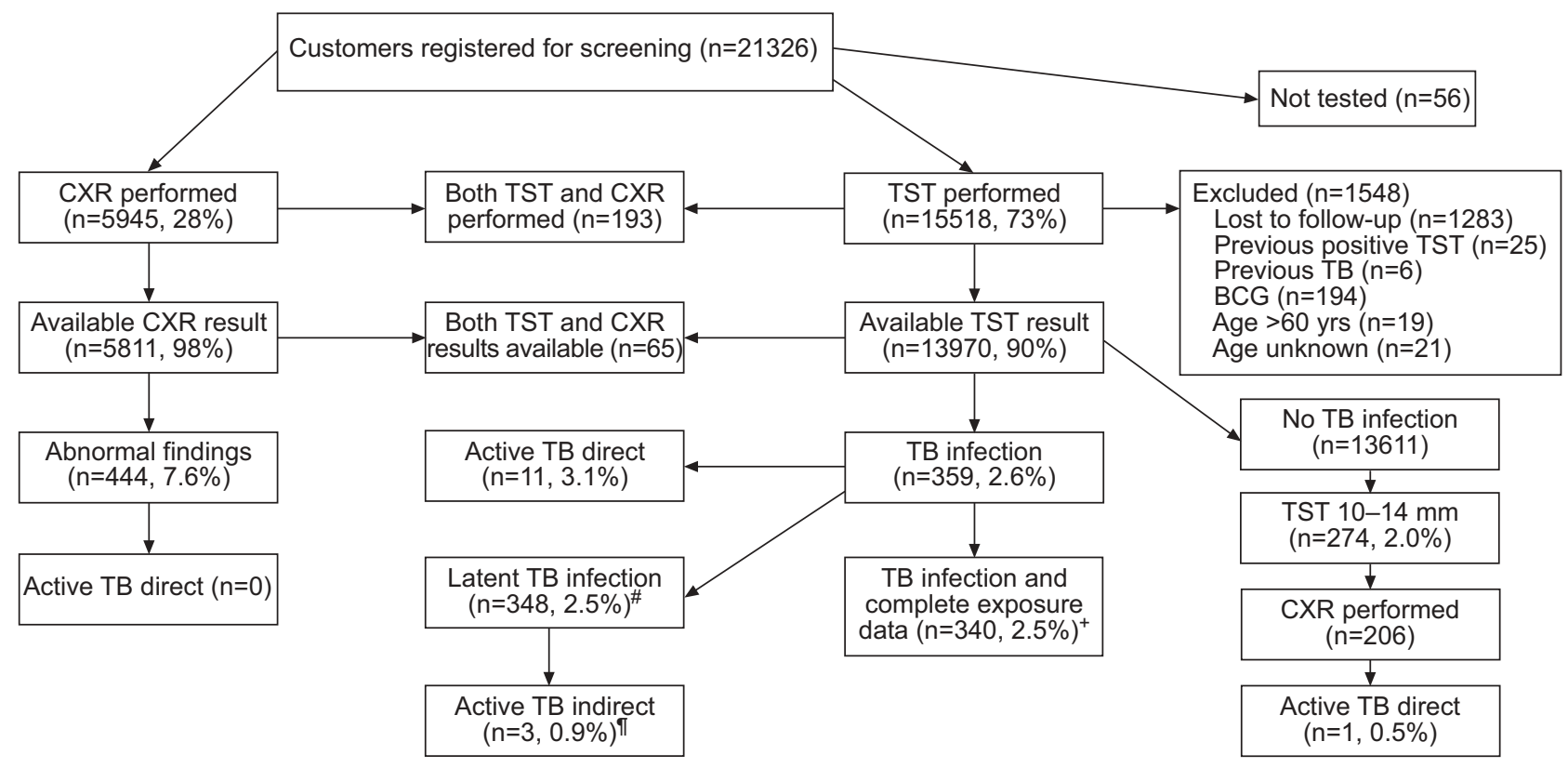

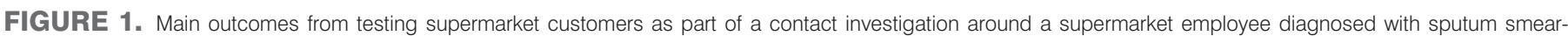

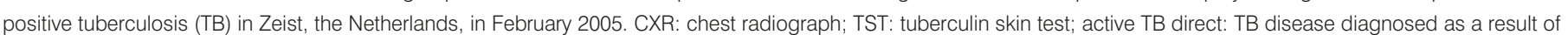

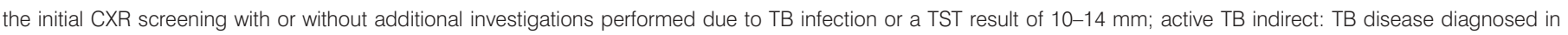

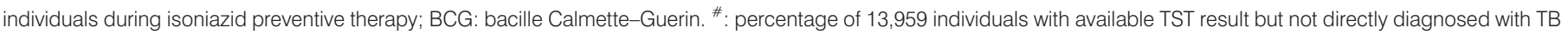
disease; ${ }^{\bullet}: 0.8 \%$ of 359 cases of TB infection; ${ }^{+}$: percentage of 13,343 individuals with available TST result and information on frequency of supermarket visits. 


\begin{tabular}{|c|c|c|c|}
\hline \multirow[t]{2}{*}{ TABLE 1} & \multicolumn{3}{|c|}{$\begin{array}{l}\text { Characteristics of } 19,716 \text { supermarket } \\
\text { customers exposed to an infectious tuberculosis } \\
\text { (TB) patient, by availability of tuberculin skin test } \\
\text { (TST) or initial chest radiograph (CXR) results }\end{array}$} \\
\hline & & TST & CXR \\
\hline \multicolumn{2}{|l|}{ Total } & $13970(100)$ & $5811(100)$ \\
\hline \multicolumn{2}{|l|}{ Male } & $6142(44.0)$ & $2592(44.6)$ \\
\hline \multicolumn{4}{|l|}{ Age yrs } \\
\hline \multicolumn{2}{|l|}{$<5$} & $1143(8.1)$ & $29(0.5)$ \\
\hline \multicolumn{2}{|l|}{$5-14$} & 2140 (15.3) & $114(2.0)$ \\
\hline \multicolumn{2}{|l|}{$15-24$} & $2062(14.8)$ & $240(4.1)$ \\
\hline \multicolumn{2}{|l|}{$25-34$} & $2519(18.0)$ & $445(7.7)$ \\
\hline \multicolumn{2}{|l|}{$35-44$} & $2771(19.8)$ & $553(9.5)$ \\
\hline \multicolumn{2}{|l|}{$45-54$} & 2260 (16.2) & $684(11.8)$ \\
\hline \multicolumn{2}{|l|}{$55-64$} & $1075(7.7)$ & $1418(24.4)$ \\
\hline \multicolumn{2}{|l|}{$>64$} & & $2313(39.8)$ \\
\hline \multicolumn{2}{|l|}{ Dutch-born } & $13355(95.6)$ & $4057(69.8)$ \\
\hline \multicolumn{2}{|c|}{ Previous BCG ${ }^{\#}$} & & $1350(23.2)$ \\
\hline \multicolumn{2}{|c|}{ Previous TB diagnosis } & & $193(3.3)$ \\
\hline \multicolumn{2}{|c|}{ Previous positive TST } & & $624(10.7)$ \\
\hline \multicolumn{2}{|c|}{ Immunocompromising condition } & $191(1.4)$ & $253(4.4)$ \\
\hline
\end{tabular}

Data are presented as $n(\%)$. Due to missing values, data may not add up to the totals. BCG: bacille Calmette-Guerin. ${ }^{\#}$ : BCG vaccine reported after first year of life.

$100,000)$. The remaining three cases were classified as indirect yield (14 per 100,000 ; $95 \%$ CI $4-45$ per 100,000; table 2). All initial diagnoses of TB without culture result or with negative cultures were confirmed by independent clinical review.

Information on frequency of visits to the supermarket was obtained from 13,343 (96\%) of the 13,970 individuals with a
TST result. The prevalence of TBI was twice as high in customers visiting the supermarket on average at least twice a week as in those visiting less than once a month (adjusted OR 2.1, 95\% CI (1.6-2.9); table 3). Compared with individuals with a TST reaction of $0-4 \mathrm{~mm}$, the proportion of individuals with a TST $\geqslant 15 \mathrm{~mm}$ or $10-14 \mathrm{~mm}$ increased with the frequency of supermarket visits (Chi-squared test $\mathrm{p}<0.001$ and $\mathrm{p}=0.011$, respectively).

The age-stratified background prevalence of remote infections among the customers screened by TST, as estimated from the historical reference data, was $1.5 \%$, and the overall prevalence $(95 \% \mathrm{CI})$ of TBI diagnosed in the present investigation was 2.5 (2.3-2.8)\%. Among the 340 TBI cases who reported data on supermarket visits, the estimated proportion of remote infections was 58 (53-64)\% (198 out of 340; table 4). This proportion was $56(44-71) \%$ when based on the average AR. After adjustment for false-positive TST reactions, the estimated number of detected cases of true recent TBI was 117, for an overall prevalence of $0.9 \%$ and an estimated proportion of true positive and recently acquired TBI of $34 \%$ (table 4 ). The overall number needed to be tested by TST, in order to identify one true recent TBI, was 114. These indicators varied strongly with frequency of supermarket visits (table 4). Among customers who reported at least two supermarket visits per week, the prevalence of detected true recent TBI was $2.3 \%$, the estimated proportion of TBI that was true positive and recently acquired was $58 \%$, and the number of customers needed to be tested in order to identify one true case of recently acquired TBI was 43 . Among customers who reported one visit per month or fewer, these figures were $<0.1 \%, 1 \%$ and 4,148 , respectively.

\section{DISCUSSION}

The present extensive contact investigation of $>21,000$ supermarket customers resulted in early detection and treatment of 15 TB patients and identification of 348 cases of LTBI. Despite

TABLE 2 Characteristics of the 15 cases of tuberculosis (TB) disease diagnosed as a result of the contact investigation

\begin{tabular}{|c|c|c|c|c|c|c|c|}
\hline Sex & Age yrs & Symptoms & Radiographical findings & M. tuberculosis culture & RFLP pattern ${ }^{\#}$ & Yield & Remarks \\
\hline Male & 15 & None & $\mathrm{HL}$ & ND & NA & Indirect & Developed during IPT \\
\hline Female & 31 & Fatigue & HL; IL LUL & Negative & NA & Direct & \\
\hline Male & 48 & NA & NA & Positive & Same & Direct & \\
\hline Male & 2 & Cough, fever, night sweats & $\mathrm{HL}$ & ND & NA & Direct & \\
\hline Female & 14 & Cough & IL RUL & Positive & Same & Direct & \\
\hline Male & 58 & Cough & IL RUL & Negative & NA & Direct & PCR positive \\
\hline Female & 29 & Night sweats & IL RCL & Positive & Different & Direct & Different $M$. tuberculosis strain \\
\hline Female & 58 & None & IL RUL & Positive & NA & Direct & \\
\hline Female & 50 & Cough, night sweats & 2 IL LUL; PT; PE & Positive & Same & Direct & TST 10-14 mm \\
\hline Female & 49 & None & PE & Negative & NA & Direct & Pleural fluid, ZN positive \\
\hline
\end{tabular}

M. tuberculosis: Mycobacterium tuberculosis; RFLP: restriction fragment length polymorphism; ND: not done; NA: not available; HL: hilar lymphadenopathy; IL: infiltrative lesion; RUL: right upper lobe; LUL: left upper lobe; NL: nodular lesion; RCL: right central lobe; PT: pleural thickening; PE: pleural exudate; IPT: isoniazid preventive therapy; TST: tuberculin skin test; ZN: microscopic examination of Ziehl-Neelsen-stained clinical specimen. ${ }^{*}$ : the same as or different from the index case. 


\begin{tabular}{|c|c|c|c|c|}
\hline \multirow{2}{*}{$\begin{array}{l}\text { TABLE } 3 \\
\text { Variable }\end{array}$} & \multicolumn{4}{|c|}{$\begin{array}{l}\text { Association between prevalence of tuberculosis } \\
\text { infection (TBI) and potential risk factors for } \\
\text { infection among supermarket customers }\end{array}$} \\
\hline & & TBI prevalence ${ }^{\#}$ & OR $(95 \% \mathrm{Cl})^{\circ}$ & p-value ${ }^{+}$ \\
\hline \multicolumn{5}{|c|}{ Supermarket visits } \\
\hline$<1$ per mo & & $70 / 4148(1.7)$ & & \\
\hline $\begin{array}{l}<1 \text { per we } \\
\text { month }\end{array}$ & $>1$ per & $65 / 3149(2.1)$ & $1.2(0.9-1.7)$ & \\
\hline 1 per week & & 98/3352 (2.9) & $1.6(1.2-2.2)$ & \\
\hline$\geqslant 2$ per we & & $107 / 2694(4.0)$ & $2.1(1.6-2.9)$ & $<0.001^{\S}$ \\
\hline \multicolumn{5}{|l|}{ Sex } \\
\hline Female & & 212/7542 (2.8) & & \\
\hline Male & & $144 / 6142(2.3)$ & $0.9(0.7-1.1)$ & 0.431 \\
\hline \multicolumn{5}{|l|}{ Age yrs } \\
\hline$<5$ & & $31 / 1143(2.7)$ & & \\
\hline $5-14$ & & 18/2140 (0.8) & $0.3(0.2-0.6)$ & \\
\hline $15-24$ & & 34/2062 (1.7) & $0.6(0.4-1.0)$ & \\
\hline $25-34$ & & $54 / 2519(2.1)$ & $0.8(0.5-1.3)$ & \\
\hline $35-44$ & & $81 / 2771(2.9)$ & $1.0(0.6-1.6)$ & \\
\hline $45-54$ & & 86/2260 (3.8) & $1.2(0.8-2.0)$ & \\
\hline $55-64$ & & $55 / 1075(5.1)$ & $1.7(1.1-2.8)$ & $<0.001$ \\
\hline \multicolumn{5}{|c|}{$\begin{array}{l}\text { Immunocompromising } \\
\text { condition }\end{array}$} \\
\hline No & & $342 / 13524(2.4)$ & & \\
\hline Yes & & $11 / 191(5.8)$ & $2.0(1.0-3.7)$ & 0.054 \\
\hline \multicolumn{5}{|c|}{ Country of birth } \\
\hline The Nether & nds & 338/13355 (2.5) & & \\
\hline Outside the & Vetherlands & $18 / 424(4.3)$ & $1.5(0.9-2.5)$ & 0.168 \\
\hline
\end{tabular}

this considerable yield, the current evaluation shows that, in retrospect, this investigation could have been performed in a more efficient way. No case of TB disease was found among 5,811 customers initially examined by chest radiography, and 114 customers needed to be skin tested in order to identify one true-positive case of recently acquired TBI. If treatment was indicated for recently acquired LTBI only, as is the policy in the Netherlands [18], $66 \%$ of the LTBI cases eligible for prophylactic treatment would not have needed it.

The current results support the hypothesis that the index patient was the source of a considerable number of TB infections. The identical $M$. tuberculosis DNA fingerprints in the index case and five of the seven customers with cultureconfirmed TB indicate a direct transmission link. None of the TB cases were reported to be related to each other and $80 \%$ reported at least one weekly visit to the supermarket. As 34\% of the TBI cases were estimated to have a true positive TST due to recent infection, the source patient probably transmitted $M$. tuberculosis to at least 117 supermarket customers. From a TB control perspective, early detection and treatment of previously unrecognised, culture-positive TB cases is warranted and in the present case has most likely prevented further transmission of TB to several others.
From the perspective of monetary and non-monetary costs to society and the public health system, there are several disadvantages to such a large-scale investigation. Although no data were collected to allow cost-benefit analysis, it is estimated that $€ 500,000$ were spent on this investigation. In order to detect one case of TB disease, 1,293 customers had to be investigated by TST. Moreover, the present authors estimated that $56-58 \%$ of the detected TBI cases were due to remote infection and, thus, most likely unrelated to exposure in the supermarket. The estimated lifetime probability of progression from LTBI to TB disease without preventive treatment is $5-15 \%$, of which $55-85 \%$ is estimated to occur in the first 5 yrs after infection; both depend on age at infection [24]. Thus, with remote LTBI, this probability is low unless the individual becomes immunocompromised [25]. It was estimated that $25(18 \%)$ out of 142 recent TBI cases had falsepositive TSTs, most likely due to cross-reactions with nontuberculous mycobacteria. This was despite a specificity of $99.8 \%$ for the TST cut-off of $15 \mathrm{~mm}$ applied for immunocompetent customers aged $>5$ yrs [15]. This illustrates the phenomenon that, even with high specificity, the positive predictive value of a test will be affected when the prevalence of infection is low [26].

The TST cut-off for LTBI of $15 \mathrm{~mm}$, which was used in the present contact investigation, follows Dutch guidelines [18]. Different cut-off values are recommended for different target populations according to their expected risk of recent infection, and a cut-off of $10 \mathrm{~mm}$ is used for immunocompetent individuals with documented contact with an infectious TB patient. In the periphery of a large contact investigation a cut-off of $15 \mathrm{~mm}$ is advised. With the low prevalence of LTBI in the present study population, a cut-off of $10 \mathrm{~mm}$ would have given a higher sensitivity for detecting LTBI but a lower specificity, resulting in more false-positive TSTs [15]. For screening for TB disease, a cut-off of $10 \mathrm{~mm}$ was nonetheless adopted, which yielded one additional case of TB disease. The prevalence of TB disease in this group $(0.5 \%)$ was, however, lower than that among individuals with TST reactions $\geqslant 15 \mathrm{~mm}(3.9 \%$; $=0.003)$.

The present study has limitations. Categorised frequency of visits to the supermarket was used as a proxy for exposure to the index case and this might be prone to recall bias. Furthermore, no data were collected on socioeconomic status, which could have confounded the findings.

The estimates of recent TBI were extrapolated from infection prevalence measured among male military conscripts from 1910-1980. They do not take into account possible differences in infection prevalence among females, and assumptions were made about the infection prevalence for age groups born after the last age cohort that had been included in the military screening programme. Nonetheless, the present estimate of the background prevalence of remote infection $(1.48 \%)$ seems robust, since it is close to the observed prevalence of infection in the lowest exposure category $(70(1.69 \%)$ out of 4,148$)$. The difference indicates underestimation of the background prevalence by only $0.21 \%$. The resulting bias would only decrease the proportion of infections that were due to transmission in the supermarket, thereby reinforcing the conclusion that the present investigation was inefficient in finding cases of recent LTBI.

Expansion of the present contact investigation from occupational contacts to supermarket customers could have been 


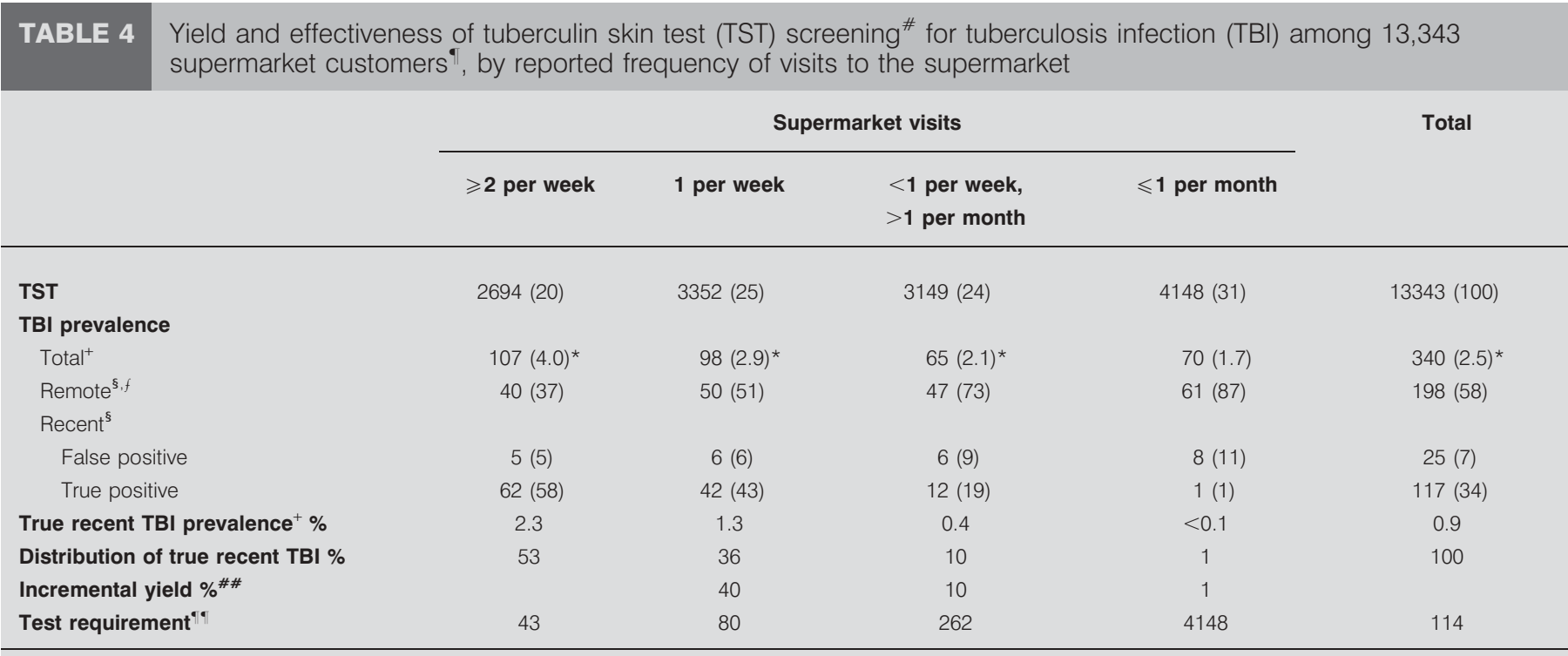

Data are presented as $n(\%)$, unless otherwise stated. ${ }^{*}$ : sensitivity and specificity of TST are $79.8 \%$ and $99.8 \%$, respectively [15]; ": for 629 (4.5\%) individuals with an available TST result, including 19 with positive TST, no exposure data were available; ${ }^{+}: \%$ of TST; ${ }^{\varsigma}$ : \% of total TBI; ${ }^{\text {: }}$ expected age-adjusted prevalence of remote TBI, 0.0148 , multiplied by number of TST; ${ }^{\# \#}$ : proportion of true recent TBI identified additionally by screening in supermarket visit category indicated; ${ }^{*}$ : number of subjects needed to be tested in order to identify one true recent TBI case, TST divided by true recent TBI. *: $p<0.05$ for comparison with expected age-adjusted prevalence of remote TBI of $1.48 \%$.

done more effectively. First, radiographical screening without prior testing for TBI could have been omitted. Secondly, testing could have been more selective without affecting the public health benefits. If skin testing had initially been limited to the customers who visited the supermarket at least once a week, more than half of the customers would not have been tested, while $83 \%$ of the cases of TB disease and $89 \%$ of the true recent TBI cases would have been detected. Half of the cases identified as TBI would have been true positive and recent, and the number of individuals needed to be tested in order to find one case of true recent TBI would have been halved from 114 to 58. Interim analysis would then have suggested that further expansion of the investigation to customers with less exposure would result in low effectiveness. This approach would also have reduced the costs considerably, although acceptance of, and compliance with, such a selective approach needs to be tested.

Thirdly, interferon- $\gamma$ release assays (IGRA) could have been used instead of or in addition to the TST, to improve the positive predictive value and allow testing for TBI of customers with a history of BCG vaccination [27]. Recent studies suggest that IGRA may even be more specific for recent than for remote infections [28, 29].

Finally, an awareness-raising campaign among general practitioners and medical specialists might have led to early detection and treatment of many of the recent TB cases and could be considered as an alternative to such a large contact investigation. The ethical implications of waiting for TB cases to occur must, however, be considered. A structured protocol for large-scale TB contact investigation is highly warranted in the Netherlands and is currently under development.

In conclusion, the present study has two major implications. First, any rational and efficient approach to contact investigation will identify only a fraction of transmissions accomplished by a tuberculosis source patient who had the opportunity to expose large numbers of individuals. Secondly, the optimal size of a contact investigation is setting specific and, apart from available resources and logistical considerations, depends on the background prevalence of latent tuberculosis infection among the contacts. The estimated number of intensely exposed contacts that needed to be screened to identify one case of true recent infection can be a useful indicator for guiding the decision to expand an investigation to less-exposed contacts.

\section{ACKNOWLEDGEMENTS}

The authors gratefully acknowledge the staff at the Municipal Health Services Midden-Nederland and Utrecht Region (the Netherlands), other personnel of the Municipality of Zeist (the Netherlands) and all volunteers who contributed to the investigation. The authors would like to thank S. van der Plas (National Institute for Public Health and the Environment, Bilthoven, the Netherlands), A. Bosman (European Center for Disease Prevention and Control, Stockholm, Sweden) and M. Valenciano (Instituto de Salud Carlos III, Madrid, Spain) for support in data management and critical review of earlier drafts; H. de Lange and G. Doornenbal (both Municipal Health Services (GG and GD) Utrecht, Utrecht, the Netherlands) for logistics and administrative matters; and W. de Lange (University Lung Center Dekkerswald, Groesbeek, the Netherlands) for clinical review of the culture-negative TB cases.

The Municipality of Zeist was involved in design and conduct of the present investigation but had no role in the collection, management, analysis or interpretation of the data, nor in the preparation, review or approval of the manuscript. 


\section{REFERENCES}

1 Global Tuberculosis Control-Surveillance, Planning, Financing. WHO Report 2007 (WHO/HTM/TB/2007.376). Geneva, World Health Organization, 2007.

2 Broekmans JF, Migliori GB, Rieder HL, et al. European framework for tuberculosis control and elimination in countries with a low incidence. Recommendations of the World Health Organization (WHO), International Union Against Tuberculosis and Lung Disease (IUATLD) and Royal Netherlands Tuberculosis Association (KNCV) Working Group. Eur Respir J 2002; 19: 765-775.

3 Targeted tuberculin testing and treatment of latent tuberculosis infection. American Thoracic Society. MMWR Recomm Rep 2000; 49: 1-51.

4 Dasgupta K, Schwartzman K, Marchand R, Tennenbaum TN, Brassard P, Menzies D. Comparison of cost-effectiveness of tuberculosis screening of close contacts and foreign-born populations. Am J Respir Crit Care Med 2000; 162: 2079-2086.

5 Etkind SC, Veen J. Contact follow-up in high- and lowprevalence countries. In: Reichman LB, Hershfield ES, eds. Tuberculosis: A Comprehensive International Approach. 2nd Edn. New York, Marcel Dekker Inc., 2000; pp. 377-396.

6 Marks SM, Taylor Z, Qualls NL, Shrestha-Kuwahara RJ, Wilce MA, Nguyen $\mathrm{CH}$. Outcomes of contact investigations of infectious tuberculosis patients. Am J Respir Crit Care Med 2000; 162: 2033-2038.

7 Reichler MR, Reves R, Bur S, et al. Evaluation of investigations conducted to detect and prevent transmission of tuberculosis. JAMA 2002; 287: 991-995.

8 Jereb J, Etkind SC, Joglar OT, Moore M, Taylor Z. Tuberculosis contact investigations: outcomes in selected areas of the United States, 1999. Int J Tuberc Lung Dis 2003; 7: Suppl. 3, S384-S390.

9 National Tuberculosis Controllers Association, Centers for Disease Control and Prevention (CDC). Guidelines for the investigation of contacts of persons with infectious tuberculosis. Recommendations from the National Tuberculosis Controllers Association and CDC. MMWR Recomm Rep 2005; 54: 1-47.

10 Veen J. Microepidemics of tuberculosis: the stone-in-thepond principle. Tuber Lung Dis 1992; 73: 73-76.

11 Kline SE, Hedemark LL, Davies SF. Outbreak of tuberculosis among regular patrons of a neighborhood bar. $N$ Engl J Med 1995; 333: 222-227.

12 Comstock GW, Livesay VT, Woolpert SF. The prognosis of a positive tuberculin reaction in childhood and adolescence. Am J Epidemiol 1974; 99: 131-138.

13 Styblo K, Broekmans J, Borgdorff M. Expected decrease in the tuberculosis incidence during the elimination phase: how to determine its trend? Tuberculosis Surveillance and Research Unit report. Seoul, Royal Netherlands Tuberculosis Association, 1997.

14 Menzies D. What does tuberculin reactivity after bacille Calmette-Guérin vaccination tell us? Clin Infect Dis 2000; 31: Suppl. 3, S71-S74.
15 Berkel GM, Cobelens FG, de Vries G, Draayer-Jansen IW, Borgdorff MW. Tuberculin skin test: estimation of positive and negative predictive values from routine data. Int $J$ Tuberc Lung Dis 2005; 9: 310-316.

16 Comstock GW, Edwards LB, Philip RN, Winn WA. A comparison in the United States of America of two tuberculins, PPD-S and RT23. Bull World Health Organ 1964; 31: 161-170.

17 The WHO standard tuberculin test. WHO/TB/Technical Guide/3. Geneva, World Health Organization, 1963.

18 Lambregts-van Weezenbeek CS, Cobelens FG, Mensen EA, Commissie voor Praktische Tuberculosebestrijding. [The tuberculin skin test in the Netherlands: new policies for an old test; guideline from the Netherlands Tuberculosis Control Policy Committee]. Ned Tijdschr Geneeskd 2003; 147: 543-546.

19 van Embden JD, Cave MD, Crawford JT, et al. Strain identification of Mycobacterium tuberculosis by DNA fingerprinting: recommendations for a standardized methodology. J Clin Microbiol 1993; 31: 406-409.

20 van Soolingen D, de Haas PEW, Kremer K. Restriction fragment length polymorphism typing of mycobacteria. In: Parish T, Stoker NG, eds. Mycobacterium Tuberculosis Protocols. Totowa, Humana Press Inc., 2001; pp. 165-203.

21 Hosmer DW, Lemeshow S. Applied Logistic Regression. New York, John Wiley \& Sons, 1989.

22 Guld J, Bentzon MW, Bleiker MA, Griep WA, Magnusson M, Waaler H. Standardization of a new batch of purified tuberculin (PPD) intended for international use. Bull World Health Organ 1958; 19: 845-951.

23 Rockhill B, Newman B, Weinberg C. Use and misuse of population attributable fractions. Am J Public Health 1998; 88: $15-19$.

24 Vynnycky E, Fine PE. Lifetime risks, incubation period, and serial interval of tuberculosis. Am J Epidemiol 2000; 152: 247-263.

25 Horsburgh CR Jr. Priorities for the treatment of latent tuberculosis infection in the United States. $N$ Engl J Med 2004; 350: 2060-2067.

26 Hennekens $\mathrm{CH}$, Buring JE. Epidemiology in Medicine. Philadelphia, Lippincott Williams \& Wilkins, 1987; pp. 336-339.

27 Menzies D, Pai M, Comstock G. Meta-analysis: new tests for the diagnosis of latent tuberculosis infection: areas of uncertainty and recommendations for research. Ann Intern Med 2007; 146: 340-354.

28 Ewer K, Millington KA, Deeks JJ, Alvarez L, Bryant G, Lalvani A. Dynamic antigen-specific T-cell responses after point-source exposure to Mycobacterium tuberculosis. Am J Respir Crit Care Med 2006; 174: 831-839.

29 Arend SM, Thijsen SF, Leyten EM, et al. Comparison of two interferon- $\gamma$ assays and tuberculin skin test for tracing tuberculosis contacts. Am J Respir Crit Care Med 2007; 175: 618-627. 